\title{
La Enseñanza pre-Universitaria en Ciencia y Tecnología Puede Influir en las Funciones Ejecutivas
}

\author{
Marta Méndez ${ }^{1}$, Natalia Arias ${ }^{1}$, José R. Menéndez ${ }^{2}$, \\ José R. Villar ${ }^{3}$, Ángel Neira ${ }^{3}$, Pedro V. Romano ${ }^{3}$, \\ José Carlos Núñez ${ }^{4}$, Jorge L. Arias ${ }^{1}$
}

${ }^{1}$ Departamento de Psicología, Instituto de Neurociencias del Principado de Asturias (INEUROPA), Universidad de Oviedo, Oviedo, España

${ }^{2}$ Departamento de Física, Universidad de Oviedo, Oviedo, España ${ }^{3}$ Departamento de Informática, Universidad de Oviedo, Gijón, España

${ }^{4}$ Departamento de Psicología, Universidad de Oviedo, Oviedo, España

España

Correspondencia: Marta Méndez. Facultad de Psicología, Plaza Feijoo s/n, 33003, Oviedo. España. E-mail: mendezlmarta@uniovi.es

(C) Education \& Psychology I+D+i and Ilustre Colegio Oficial de la Psicología de Andalucía Oriental 


\section{Resumen}

Introducción. Las áreas científicas y tecnológicas incluyen una enseñanza basada en una alta especialización viso-espacial y de resolución de problemas. Estas capacidades espaciales y de resolución de problemas están integradas en un plan de estudios que promueve la comprensión de asignaturas científicas y técnicas y están relacionadas con el desarrollo de las funciones ejecutivas (FE). Nuestro objetivo es evaluar si los estudiantes que han estudiado las ramas de Ciencia y Tecnología en los estudios pre-universitarios presentan mejores FE implicadas en el razonamiento espacial y la resolución de problemas.

Método. Se evaluaron seis FE diferentes en dos grupos de estudiantes: estudiantes de Ciencia y Tecnología (grupo ST) y estudiantes matriculados en el primer curso de Psicología con estudios pre-universitario no orientados hacia la ciencia y la tecnología (grupo No-ST). Evaluamos la memoria de trabajo espacial, la planificación, la inhibición, la flexibilidad mental y el razonamiento verbal y abstracto en ambos grupos.

Resultados. El estudio reveló que la memoria espacial y la planificación fueron significativamente mejores en el grupo ST en comparación con el grupo No-ST. El grupo No- ST presenta más impulsividad. No hubo diferencias entre los grupos en el resto de los FE evaluadas.

Conclusión. Los estudios pre-universitarios de ciencia y tecnología pueden influir en las FE mediante la mejora de la planificación y la memoria de trabajo espacial, así como en una menor impulsividad.

Palabras Clave: ingeniería, memoria de trabajo espacial, planificación, inhibición, flexibilidad mental. 


\title{
Pre-University Tuition in Science and Technology can Influence Executive Functions
}

\begin{abstract}
Introduction. Scientific and technological areas include tuition based on highly visuo-spatial specialization and problem solving. Spatial skills and problem solving are embedded in a curriculum that promotes understanding of Science and technical subjects. These abilities are related to the development of executive functions (EFs). We aim to assess whether students who have studied Science and Technology branches in the High School present improved EFs involved in spatial reasoning and problem solving.
\end{abstract}

Method. We assessed six different EFs in two groups of students: Science and Technology students (ST group) and students enrolled in the first course of Psychology whose preuniversity studies were not oriented towards science and technology (No-ST group). The EFs tests that we applied in the ST and No-ST groups assessed spatial working memory, planning, inhibition, mental flexibility, verbal and abstract reasoning.

Results. The study revealed that spatial working memory and planning were significantly better in the ST group comparing with the No-ST group. The No-ST group presented more impulsivity. There were no differences between the groups in the rest of EFs assessed.

Conclusion: Pre-university studies that include Science and Technology subjects can influence EFs by improving planning and spatial working memory, as well as leading to less impulsivity.

Keywords: Engineering; Spatial working memory; Planning; Inhibition; Mental Flexibility. 


\section{Introducción}

Las áreas científicas y tecnológicas, como las matemáticas, la física o la ingeniería, incluyen enseñanzas basadas en la especialización visoespacial y la solución de problemas. De hecho, las habilidades espaciales y la resolución de problemas están integradas en un plan de estudios que promueve una comprensión holística de materias científicas y técnicas (Marunic \& Glazar, 2012). Los investigadores de ciencias indican que la capacidad espacial es necesaria para tener éxito en dominios específicos, como las matemáticas, así como en la arquitectura, la cartografía, la química, la física y la ingeniería (Diezmann \& Lowrie, 2012; Coleman \& Gotch, 1998). Por lo tanto, una gran parte de la investigación en ciencia y matemáticas se centra en cómo las habilidades espaciales pueden predecir el éxito de los estudiantes en la comprensión de determinados conceptos (Sorby, 2001). Junto con la capacidad espacial, la resolución de problemas es una habilidad esencial para tener éxito en las asignaturas de ciencia y matemáticas. Esta capacidad incluye la planificación, diseño, construcción, evaluación y rediseño de una solución para un problema dado en cualquier disciplina (Stamovlasis \& Tsaparlis, 2005; Lawson, 2004). Estos procesos que permiten la planificación, control y seguimiento de comportamientos complejos dirigidos a una meta se suelen denominar funciones ejecutivas (FE) (Seiferth, Thienel, \& Kirchner, 2007).

Las FE son muy importantes porque median los procesos de aprendizaje (St. ClairThompson, Stevens, Hung, \& Bolder, 2010; Rabin, Fogel, \& Nutter-Upham, 2010). Por lo tanto, estas FE se entienden como capacidades cognitivas específicas o habilidades de orden superior que influyen en el rendimiento en otras tareas cognitivas. Estas habilidades cognitivas superiores incluyen, pero no se limitan a, la flexibilidad mental (la capacidad de mantener y modificar la conducta), la planificación (la capacidad de gestionar la demanda de trabajo en una secuencia organizada de conductas), la inhibición de respuesta (la capacidad de detener la propia conducta en el momento adecuado), la memoria de trabajo (la capacidad de mantener y manipular la información en la mente), las habilidades de organización, el razonamiento, la resolución de problemas y el pensamiento abstracto (la capacidad de llegar a conclusiones a través del uso de símbolos o generalizaciones) (Alvarez \& Emory, 2006).

Aunque, inicialmente, la corteza frontal fue entendida como un módulo concreto donde las diferentes FE residían de forma aislada (Luria, 2002). Hoy en día, cada vez está más 
claro que las FE son apoyadas por varias áreas del cerebro con las cuáles forman redes funcionales complejas (Enríquez-Geppert, Huster, \& Herrmann, 2013).

Algunas de estas FE pueden ser moduladas por el tipo de instrucción a la que estamos expuestos. Se ha demostrado que la experiencia en tareas espaciales, común en la ingeniería y la ciencia, tiene un gran impacto en el desarrollo de la memoria de trabajo espacial. De hecho, varios experimentos han demostrado que las aplicaciones diseñadas para mejorar la capacidad espacial tienen el efecto de aumentar la memoria de trabajo espacial en los estudiantes de ingeniería y ciencia, así como en la población en general (Carbonell, Saorín, de la Torre, \& Marrero, 2011). En cuanto a los estudios preuniversitarios, las investigaciones señalan que es posible mejorar las habilidades espaciales y de resolución de problemas a través de la ciencia, las matemáticas y las clases de dibujo (Fantz, Siller, \& DeMiranda, 2011). Este tipo de experiencias de aprendizaje ayuda a los estudiantes a familiarizarse con nueva información a través de la resolución de problemas con el fin de recordar fácilmente nuevos conceptos y construir imágenes mentales y, por lo tanto, podría contribuir al desarrollo de la planificación, la flexibilidad y la memoria de trabajo espacial.

\section{Objetivo e hipótesis}

Nuestro objetivo es evaluar si los estudiantes que han estudiado las ramas de Ciencia y Tecnología en secundaria y están matriculados en el primer curso de sus estudios universitarios presentan mejores FE relacionadas con el razonamiento espacial y la resolución de problemas que los estudiantes que no recibieron esta formación tecnológica específica. Para ello, evaluamos seis FE diferentes en estudiantes de ciencia y tecnología y alumnos matriculados en el primer curso de Psicología cuyos estudios preuniversitarios no estaban orientados hacia la ciencia y la tecnología. Las FE que evaluamos fueron la memoria espacial de trabajo, la planificación, la inhibición, la flexibilidad mental, el razonamiento verbal y el razonamiento abstracto. Basándonos en los conocimientos previos, se hipotetizó que los estudiantes de Ciencia y Tecnología presentarían una mejor capacidad de memoria de trabajo y planificación que los otros estudiantes. 


\section{Método}

\section{Participantes}

Ciento cuarenta alumnos (86 mujeres y 54 varones, edad media $=19,10$ años DST= 1,25), todos ellos estudiantes de primer curso en la Universidad de Oviedo (España), participaron en el estudio a cambio de 1 crédito. Todos los participantes poseían una visión normal o corregida. El estudio se realizó con 57 estudiantes que estudiaron la rama de Ciencia y Tecnología en la Escuela Secundaria (Grupo ST) y 83 estudiantes de Psicología, cuyos estudios preuniversitarios no estaban orientados hacia la ciencia y la tecnología (Grupo No-ST). Todos los procedimientos y medidas fueron aprobados por el Comité de Ética de la Universidad de Oviedo.

\section{Medidas}

\section{Memoria de Trabajo Espacial}

Para testar la memoria de trabajo espacial, se utilizó la adaptación española de la prueba Rotación Mental de figuras macizas (RFM) (Yela, 1968; Thurstone \& Thurstone, 1949), en la que se evalúa la capacidad para rotar mentalmente figuras sólidas. La prueba consta de 21 ítems, en cada uno se presenta una figura sólida tridimensional compleja y cinco figuras de elección. En cada ítem, el participante tiene que elegir cuál de las figuras a elegir representa una forma que coincide con la muestra girada mentalmente. Existe un tiempo límite de 5 minutos para la solución de esta prueba. Se registró el número de respuestas correctas.

\section{Flexibilidad Mental}

Se utilizó la prueba de Clasificación de Cartas de Wisconsin (WCST) (Heaton, Chelune, Talley, Kay, \& Curtiss, 1993) para evaluar la flexibilidad mental, la capacidad de mostrar flexibilidad ante el cambio de programas de reforzamiento. Se utilizó una versión computarizada de la tarea. Inicialmente, se mostraron un número de cartas estímulo al participante. Se les indicó a los participantes que su tarea consistía en emparejar las cartas de forma exitosa, pero no se les dijo el criterio exitoso de clasificación; sin embargo, se les indicaba si un emparejamiento particular era un acierto o fallo. La prueba incluyó 64 ensayos. Se registró el número de emparejamientos correctos. 


\section{Planificación}

Se utilizó una versión computarizada de la Torre de Hanoi (TOH) (Welsh, 1991). Evaluamos la ejecución de los sujetos en la versión de la tarea compuesta por cinco discos. La tarea consiste en tres barras y cinco discos de tamaño gradualmente menor. El objetivo de la tarea es mover toda la pila de discos a otra varilla, siguiendo las siguientes reglas: sólo es posible mover un disco en cada movimiento, cada movimiento consiste en tomar el disco superior de una de las varillas y deslizarlo a otra varilla para colocarlo en la parte superior de los otros discos que ya pueden estar en la varilla. Además, no se puede colocar un disco mayor sobre otro menor. La planificación es un componente clave de las habilidades de solución de problemas necesarias para lograr este objetivo. Se registraron el número de movimientos de discos realizados por los participantes y el tiempo total empleado en la tarea.

\section{Inhibición}

El control inhibitorio requiere detener una respuesta cuando sea necesario. Esta FE se evaluó mediante una tarea de acción / inhibición Go-No Go que requiere que los participantes sean capaces de responder (Ir o Go) a un tipo de estímulo con una acción motora y de retener una respuesta (No Ir o No Go) a otro tipo de estímulo (Bruin, Wijers, \& van Staveren, 2001). Por lo tanto, los participantes realizan una decisión binaria ante cada estímulo que se presenta. Después de una serie de ensayos, el estímulo que necesita una respuesta motora se intercambia con el otro. En la tarea se utilizaron dos estímulos que aparecían en la pantalla del ordenador en un orden al azar: un pato y un ratón. Durante los primeros 16 ensayos, pedimos a los participantes que pulsaran la barra espaciadora del teclado cuando el pato aparecía en la pantalla del ordenador, mientras que en los últimos 16 ensayos debían presionar cuando se presentaba el ratón. Se registró el número de respuestas correctas (respuestas motoras a los estímulos correctos) y los errores de inhibición de respuesta, errores de comisión (respuestas motoras a los estímulos incorrectos).

\section{Razonamiento Verbal}

El razonamiento verbal fue evaluado mediante el Test de Aptitudes diferenciales (DAT-5) (Bennett, Seashore, \& Wesman, 2000). La prueba evalúa la capacidad de comprensión de conceptos. Los participantes deben encontrar puntos en común entre los diferentes conceptos y manipular las ideas en un nivel abstracto. Los estudiantes respondieron a 40 preguntas sobre analogías verbales en un límite de tiempo de 20 min. Se registró el número de respuestas correctas. 


\section{Razonamiento Abstracto}

El razonamiento abstracto se evaluó mediante la prueba Matrices Progresivas de Raven (Raven, Court, \& Raven, 1994) Set I y II Set. Esta prueba nos ofrece una medida del cociente de inteligencia general $(\mathrm{CI})$ de los participantes en el estudio. Se registró el número de respuestas correctas.

\section{Procedimiento}

Las pruebas se administraron en el orden descrito anteriormente.

\section{Análisis de datos}

Los datos fueron analizados mediante el paquete estadístico para las Ciencias Sociales (SPSS) versión 19. Para examinar las diferencias entre los dos grupos de nuestro estudio en cada FE, hemos comparado los grupos ST y No-ST mediante una prueba t para muestras independientes. De esta manera, pudimos comparar las FE de los estudiantes que estaban orientadas a la ciencia y la tecnología en sus estudios preuniversitarios frente a los que no lo estaban.

\section{Resultados}

De acuerdo con nuestra hipótesis, los estudiantes de Ciencia y Tecnología (grupo ST) presentan una mejor capacidad de memoria de trabajo espacial y planificación. Este grupo de estudiantes presentó mayor puntuación en la prueba de RFM que el grupo No-ST $\left(t_{138}=4.40\right.$, $p<0.001)$. Además, el grupo ST realiza menos movimientos en el test de planificación que el grupo no-ST $\left(t_{138}=3.43, p<0.001\right)$. En consecuencia, el grupo ST tardó menos tiempo en resolver la tarea $\left(t_{138}=3.27, p=0.001\right)$. Además, la capacidad de inhibición es mejor en el grupo ST. Aunque no hubo diferencias entre los grupos en el número de respuestas motoras a los estímulos correctos en la tarea Go- No Go $\left(t_{138}=0.25, p=0.80\right)$, el grupo No-ST realizó un mayor número de errores de comisión, esto es respuestas motoras al estímulo incorrecto, que el grupo ST $\left(t_{138}=2.29, p=0.02\right)$. Véase la Tabla 1. 
Tabla 1. Media y EEM de las variables medidas en ambos grupos.

\begin{tabular}{lccccc}
\hline & \multicolumn{3}{c}{ Grupo ST } & \multicolumn{2}{c}{ Grupo No-ST } \\
\cline { 2 - 5 } & Media & EEM & Media & EEM & $p$ \\
\hline Memoria de Trabajo Espacial, aciertos RFM & 10,00 & 0,58 & 7,00 & 0,39 & $<0,001^{*}$ \\
Flexibilidad Mental, aciertos WCST & 39,26 & 1,28 & 40,71 & 1,20 & 0,42 \\
Planificación, Movimientos TOH & 63,44 & 3,78 & 92,01 & 6,39 & $<0,001^{*}$ \\
Planificación, Tiempo (segundos) TOH & 153,79 & 12,69 & 225,39 & 15,92 & $0,001^{*}$ \\
Inhibición, aciertos Go-No Go & 29,82 & 0,81 & 30,07 & 0,58 & 0,80 \\
Inhibición, errores de comisión Go-No Go & 0,28 & 0,07 & 0,59 & 0,10 & $0,02^{*}$ \\
Razonamiento Verbal, aciertos DAT & 6,197 & 0,19 & 6,09 & 0,15 & 0,66 \\
Razonamiento Abstracto, aciertos Raven & 52,28 & 0,52 & 51,31 & 0,34 & 0,11 \\
\hline
\end{tabular}

*Diferencias significativas.

No hay diferencias entre los grupos en el resto de las variables evaluadas. Ambos grupos ST y No-ST presentan el mismo nivel de flexibilidad mental, ya que no se diferencian en el número de emparejamientos correctos en el WCST $\left(t_{138}=0.81, p=0.42\right)$. Además, mostraron puntuaciones similares en el razonamiento verbal, ya que el número de respuestas correctas en el DAT-5 no difirió entre los grupos $\left(t_{138}=0.44, p=0.66\right)$. Además, el número de respuestas correctas en la prueba de razonamiento abstracto fue similar entre los grupos ST y No-ST $\left(t_{138}=1.63, p=0.11\right)$. Por lo tanto, el cociente intelectual no influyó en los resultados.

\section{Discusión}

Nuestro estudio ha demostrado que los estudios preuniversitarios podrían influir en algunas FE vinculadas a la corteza prefrontal dorsolateral (CPFDL), una región del cerebro que consistentemente se ha asociado con los procesos de control cognitivo cuando incrementa la complejidad o la integración de las demandas durante el control de una determinada respuesta (Hoshi, 2006). Los estudiantes que fueron expuestos en mayor medida a asignaturas con contenidos de ciencia y tecnología mostraron una mejor memoria de trabajo espacial y planificación, así como menos impulsividad.

Las diferencias entre los grupos en la memoria de trabajo espacial pueden ser debidas a una formación específica durante la etapa de secundaria. El grupo ST cursó asignaturas obligatorias relacionadas con las matemáticas, la física y el dibujo técnico en los últimos cursos de la Escuela Secundaria. Estos temas son importantes para el desarrollo del control cognitivo y están estrechamente relacionados con las FE. La tarea específica de memoria de trabajo espacial que hemos utilizado en este estudio es una tarea de rotación mental, un compo- 
nente de la capacidad espacial (McGee, 1979). Esta tarea requiere la habilidad de manipular mentalmente, rotar, girar o invertir estímulos presentados gráficamente (Tartre, 1990). Por lo tanto, se evaluó la capacidad de rotar las imágenes mentales de los objetos, lo que refleja la capacidad de memoria de trabajo espacial. Los participantes en el estudio deben girar dos o tres objetos tridimensionales en su mente y manipular esta información espacial en varias etapas secuenciales con el fin de obtener una solución correcta (Zacks, Mires, Tversky, \& Hazeltine, 2000).

La memoria de trabajo espacial se desarrolla durante las diferentes etapas de la vida como resultado de la exposición a varios entornos de aprendizaje y experiencias (Pickering, 2001). Por lo tanto, la adquisición de habilidades espaciales complejas puede requerir de formación en ámbitos específicos como resultado de la experiencia y práctica de actividades visuoespaciales durante la instrucción preuniversitaria. Es posible que esta formación específica previa a la universidad del grupo ST mejore la capacidad de rotación mental, y, por tanto, la memoria de trabajo espacial. Por otra parte, después de la formación preuniversitaria específica en matemáticas, física y dibujo, los alumnos pueden ver facilitadas sus tasas de retención de información visual en comparación con los estudiantes que no reciben esa formación. De acuerdo con este punto de vista, diferentes estudios demuestran que el dibujo parece ser especialmente eficaz para el desarrollo de las habilidades espaciales y la formación de imágenes visuales (Sorby, 1999). Del mismo modo, O'Boyle (1998) ha demostrado que el estudio de matemáticas desarrolla la parte del cerebro involucrada en la visualización, la capacidad espacial y la memoria de trabajo de alta demanda. También se ha sugerido que la física implica una mejora específica de la memoria de trabajo (Chen y Whitehead, 2009). Además, el efecto de un entrenamiento específico de una FE puede ser transferido al procesamiento ejecutivo en general, como han demostrado Minear y Shah (2008). Estos autores proporcionan evidencia acerca de que este efecto de transferencia del entrenamiento en un dominio específico de FE no sólo se basa en una mejora no específica la automatización de procesos o control motor.

En cuanto a la planificación, esta capacidad implica el desarrollo y ejecución de planes. Es crucial para realizar de manera efectiva una amplia gama de situaciones cotidianas. La planificación implica un conjunto de procesos cognitivos que incluyen crear y ordenar objetivos, desarrollar hipótesis acerca de cómo cada uno puede ser abordado y comprobar si la consecución de uno no afecta destructivamente la consecución de otro y, si es así, revisar el procedimiento general. Además, es necesario codificar en la memoria la estructura de los pasos 
necesarios. Entonces, para ejecutar un plan es necesario seguir, recuperar de la memoria y ejecutar los distintos pasos, comprobando que el plan de acción está avanzando adecuadamente y según lo previsto (Crescentini, Seyed-Allaei, Vallesi, \& Shallice, 2012). Puede ser que la mejor capacidad de planificación presentada por el grupo ST en la prueba de TOH obedezca a su mejor memoria de trabajo para material visual. Estos dos procesos parecen estar fuertemente relacionados (Crescentini, Seyed-Allaei, Vallesi, \& Shallice, 2012). Se ha descubierto mediante técnicas de imagen funcional que la activación de la parte medial de la corteza prefrontal dorsolateral se atribuye a los componentes de memoria de trabajo de la tarea (Wagner, Koch, Reichenbach, Sauer, \& Schlosser, 2006). Además, una tarea de memoria de trabajo que requiere la retención a corto plazo y reproducción de secuencias de movimientos espaciales emparejados a los que se realizan en la tarea $\mathrm{TOH}$, activa regiones del cerebro similares a las que se activan durante la ejecución de problemas de planificación en la tarea TOH (Owen et al., 1996; Crescentini, Seyed- Allaei, Vallesi, \& Shallice, 2012).

De hecho, la CPFDL está localizada en una posición ideal para ejercer funciones de control a través de sus conexiones con múltiples regiones cerebrales proximales y distales (Cieslik et al., 2012), que posibilitan que sea propuesta como el principal efector del control ejecutivo "de arriba-abajo" (Hoshi, 2006). La evidencia acerca de la existencia de dos redes cerebrales diferentes que involucran a la CPFDL apoya nuestros datos. En particular, la activación de la red CPFDL anterior - corteza cingulada anterior está más relacionado con procesos atencionales y de inhibición, así como con las tareas que requieren la resolución de conflictos como la tarea Go / No-Go. Por el contrario, la red CPFDL posterior - corteza parietal posterior está más relacionada con la ejecución del movimiento, así como los procesos de memoria de trabajo (Cieslik et al., 2012). En este caso, el componente de memoria de esta capacidad consiste en el mantenimiento de la veracidad de la información espacial y visual en el tiempo o durante una demora, es decir, el procesamiento de una serie de transformaciones espaciales y almacenamiento de representaciones mentales en la memoria (D'Ardenne et al., 2012). Por lo tanto, se exigen capacidades de procesamiento y almacenamiento de manera simultánea, las cuales dependen de la capacidad de memoria de trabajo (Zimmer, 2008).

También hemos encontrado que los estudiantes del grupo ST mostraron un mejor control inhibitorio, cometieron menos errores de comisión, presentando menor impulsividad que el grupo No-ST. En relación con esto, una buenas habilidades de autorregulación pueden ayudar a los estudiantes a rendir de forma exitosa en matemáticas (Blair \& Razza, 2007; Badai, 
Eidelman \& Stavy, 2012). El control inhibitorio y atencional está estrechamente relacionado con la capacidad matemática en los niños, y puede predecir los resultados académicos independiente de la inteligencia general (Blair \& Razza, 2007), cuyo funcionamiento global se relaciona con la CPFDL (Barbey et al., 2012).

Curiosamente, un estudio longitudinal del desarrollo de las FE ha encontrado que las puntuaciones de tres tareas distintas de control inhibitorio, memoria de trabajo y planificación están fuertemente relacionados durante el desarrollo y pueden predecir el éxito académico (Hughes, Ensor, Wilson, \& Graham, 2010). Podría ser que los estudiantes del grupo ST de nuestro estudio representen este ejemplo. Estos estudiantes podrían haber alcanzado buenos logros en las asignaturas de ciencias como consecuencia de su capacidad más alta en los tres dominios que hemos demostrado que mejoran en este estudio: la planificación, la memoria de trabajo y el control inhibitorio. Además, a consecuencia de este mayor éxito, el entrenamiento específico en planificación y memoria de trabajo, implícito en las asignaturas de ciencias, podría también ayudar a desarrollar mejor las FE en estos dominios. Sin embargo, podría ser interesante explorar cómo el entrenamiento y transferencia de diferentes procedimientos de entrenamiento pudiera beneficiar la comprensión básica de las FE, así como su posible mejora.

\section{Agradecimientos}

Agradecemos la financiación recibida a través de las ayudas MICINN PSI 2010-19348, TIN2011-24302 y MEC AP2009-1714 para NA.

\section{Referencias}

Alvarez, J.A., \& Emory, E. (2006). Executive function and the frontal lobes: a meta-analytic review. Neuropsychological Reviews, 16(1), 17-42. DOI:10.1007/s11065-006-9002-x

Babai, R., Eidelman, R., \& Stavy, R. (2012). Preactivation of inhibitory control mechanisms hinders intuitive reasoning. International Journal of Science and Mathematics Education, 10(4), 763-775. DOI:10.1007/s10763-011-9287-y

Barbey, A.K., Colom, R., Solomon, J., Krueger, F., Forbes, C., \& Grafman, J. (2012). An integrative architecture for general intelligence and executive function revealed by lesion mapping. Brain, 135(4), 1154-1164. DOI:10.1093/brain/aws021 
Bennett, G.K., Seashore, H.G., \& Wesman, A.G. (2000). Test de aptitudes diferenciales (DAT-5) manual (Differential Aptitude Test). Madrid, Spain: TEA Editors.

Blair, C., \& Razza, R.P. (2007). Relating effortful control, executive function, and false belief understanding to emerging math and literacy ability in kindergarten. Child Development, 78(2), 647-663. DOI:10.1111/j.1467-8624.2007.01019.x

Bruin, K.J., Wijers, A.A., \& van Staveren, A.S. (2001). Response priming in a Go/Nogo task: do we have to explain the Go/Nogo $\mathrm{N} 2$ effects in terms of response activation instead of inhibition? Clinical Neurophysiology, 112, 660-1671. DOI: 10.1016/S13882457(01)00601-0

Carbonell, C., Saorín, J.L., de la Torre, J., \& Marrero, A.M. (2011). Engineers' spatial orientation ability development at the European Space for Higher Education. European Journal of Engineering Education, 36(5), 505-512. DOI:10.1080/03043797.2011.602184

Chen, W.C., \& Whitehead, R. (2009). Understanding physics in relation to working memory. Research in Science \& Technological Education, 27(2), 151-160. DOI:10.1080/02635140902853624

Cieslik, E.C., Zilles, K., Caspers, S., Roski, C., Kellermann, T.S., Jakobs, O., Eickhoff, S.B. (2012). Is there "one" DLPFC in cognitive action control? evidence for heterogeneity from co-activation-based parcellation. Cerebral Cortex. In press. DOI:10.1093/cercor/bhs256

Coleman, S.L., \& Gotch, A.J. (1998). Spatial Perception Skills of Chemistry Students. Journal of Chemical Education, 75(2), 206-209. DOI: 10.1021/ed075p206

Crescentini, C., Seyed-Allaei, S., Vallesi, A., \& Shallice, T. (2012). Two networks involved in producing and realizing plans. Neuropsychologia, 50(7), 1521-1535. DOI:10.1016/j.neuropsychologia.2012.03.005

D'Ardenne, K., Eshel, N., Luka, J., Lenartowicz, A., Nystrom, L.E., \& Cohen, J.D. (2012). Role of prefrontal cortex and the midbrain dopamine system in working memory updating. Proceedings of the Natural Academy of Science of the United States of America, 109(49), 19900-19909. DOI: 10.1073/pnas.1116727109

Diezmann, G., \& Lowrie, T. (2012). Learning to think spatially: what do students "see" in numeracy test items? International Journal of Science and Mathematics Education, 10(6),1469-1490. DOI: 10.1007/s10763-012-9350-3

Enriquez-Geppert, S., Huster, R.J., \& Herrmann, C.S. (2013). Boosting brain functions: Improving executive functions with behavioral training, neurostimulation, and 
neurofeedback. International Journal Psychophysiology, 88(1), 1-16. DOI: doi: 10.1016/j.ijpsycho.2013.02.001

Fantz, T.D., Siller, T.J., \& DeMiranda, M.A. (2011). Pre-collegiate factors influencing the self-efficacy of engineering students. Journal of Engineering Education, 100(3), 604623. DOI: 10.1002/j.2168-9830.2011.tb00028.x

Heaton, R.K., Chelune, G.J., Talley, J.L., Kay, G.G., \& Curtiss, G. (1993). Wisconsin Card Sorting Test. Odessa, FL: Psychological Assessment Resources.

Hoshi, E. (2006). Functional specialization within the dorsolateral prefrontal cortex: a review of anatomical and physiological studies of non-human primates. Neuroscience Research, 54(2), 73-84. DOI: 10.1016/j.neures.2005.10.013

Hughes, C., Ensor, R., Wilson, A., \& Graham, A. (2010). Tracking executive function across the transition to school: a latent variable approach. Developmental Neuropsychology, 35(1), 20-36. DOI: 10.1080/87565640903325691

Lawson, A. (2004). The Nature and Development of Scientific Reasoning: A Synthetic View. International Journal of Science and Mathematics Education, 2(3), 307-338. DOI:10.1007/s10763-004-3224-2

Luria, A.R. (2002). Frontal lobe syndromes. In P.J. Pinken, \& G.W. Bruyn (Eds.), Handbook of Clinical Neurology, (pp. 725-757). Amsterdam: North Holland.

Marunic, G., \& Glazar, V. (2012). Spatial ability through engineering graphics education. International Journal of Technology and Design Education, DOI:10.1007/s10798012-9211-y

McGee, M.G. (1979). Human spatial abilities: psychometric studies and environmental, genetic, hormonal and neurological influences. Psychological Bulletin, 86(5), 889-918. DOI:10.1037/0033-2909.86.5.889

Minear, M., \& Shah, P. (2008). Training and transfer effects in task switching. Memory \& Cognition, 36(8), 1470-1483. DOI:10.3758/MC.336.8.1470.

O'Boyle, M.W. (1998). On the relevance of research findings in cognitive neuroscience to educational practice. Educational Psychology Review, 10(4), 397-409. DOI:10.1023/A:1022889317826

Owen, A.M., Doyon, J., Petrides, M., \& Evans, A.C. (1996). Planning and spatial working memory: A positron emission tomography study in humans. European Journal of Neuroscience, 8, 353-364. DOI: 10.1111/j.1460-9568.1996.tb01219.x

Pickering, S.J. (2001). The development of visuo-spatial working memory. Memory, 9(4-6), 423-432. DOI:10.1080/09658210143000182 
Rabin, L.A., Fogel, J., \& Nutter-Upham, K.E. (2010). Academic procrastination in college students: the role of self-reported executive function. Journal Clinical Experimental Neuropsychology, 33(3), 344-357. DOI: 10.1080/13803395.2010.518597

Raven, J.C., Court, J.H., \& Raven, J. (1994). Raven Manual: Section 4, Advanced Progressive Matrices. Oxford Psychologists Press.

Seiferth, N.Y., Thienel, R., \& Kirchner, T. (2007). Exekutive Funstionen. In F. Scheneider, \& G.R. Fink (Eds), Funktionelle MRT in Psychiatrie und Neurologie (pp. 265-277). Berlin Heidelberg: Springer.

Sorby, S. (1999). Developing 3D spatial visualization skills. Engineering Design and Graphics Journal, 63(2), 21-32. http://www.edgj.org/index.php/EDGJ/article/viewFile/126/122

Sorby, S. (2001). Improving the spatial skills of engineering students: impact on graphics performance and retention. Engineering Design and Graphics Journal, 65(3), 31-36.

Stamovlasis, D., \& Tsaparlis, G. (2005). Cognitive Variables in Problem Solving: A Nonlinear Approach. International Journal of Science and Mathematics Education, 3(1), 7-32. DOI: $10.1007 / \mathrm{s} 10763-004-3918-5$

St. Clair-Thompson, H., Stevens, R., Hung, A., \& Bolder, E. (2010). Improving children's working memory and classroom performance. Educational Psychology, 30, 203-219. DOI: $10.1080 / 01443410903509259$

Tartre, L.A. (1990). Spatial skills, gender and mathematics. In E.H. Fennema, \& G.C. Leder (Eds), Mathematics and gender (pp. 27-59). New York NJ: Teachers College Press.

Thurstone, T.G., \& Thurstone L.L. (1949). Mechanical Aptitude II; Description of group tests. Chicago, IL: University of Chicago, Psychometric Laboratory, Report 54.

Wagner, G., Koch, K., Reichenbach, J.R., Sauer, H. R., \& Schlosser, G.M. (2006). The special involvement of the rostrolateral prefrontal cortex in planning abilities: An eventrelated fMRI study with the Tower of London paradigm. Neuropsychologia, 44, 2337 2347. DOI: 10.1016/j.neuropsychologia.2006.05.014

Welsh, M. (1991). Rule-guided behavior and self-monitoring on the Tower of Hanoi disktransfer task. Cognitive Development, 6, 59-76. DOI: 10.1016/0885-2014(91)90006-Y

Yela, M. (1968). Rotación de figuras macizas. Manual [Rotation of solid figures. Manual]. Madrid: TEA.

Zacks, J.M., Mires, J., Tversky, B., \& Hazeltine, E. (2000). Mental spatial transformations of objects and perspective. Spatial Cognition and Computation, 2, 315-332. DOI:10.1023/A:1015584100204 
Marta Méndez et al.

Zimmer, H.D. (2008). Visual and spatial working memory: from boxes to networks. Neuroscience and Biobehavioral Reviews, 32(8), 1373-139. DOI:10.1016/j.neubiorev.2008.05.016 